THE ASTROPHYSICAL JOURNAL, 532:57-64, 2000 March 20

(C) 2000. The American Astronomical Society. All rights reserved. Printed in U.S.A

\title{
NEW COSMIC MICROWAVE BACKGROUND POWER SPECTRUM CONSTRAINTS FROM MSAM1
}

\author{
G. W. Wilson, ${ }^{1,2}$ L. Knox, ${ }^{1}$ S. Dodelson, ${ }^{3}$ K. Coble, ${ }^{1}$ E. S. Cheng, ${ }^{2}$ D. A. Cottingham, ${ }^{4}$ D. J. Fixsen, ${ }^{5}$ A. B. Goldin, ${ }^{1}$ \\ C. A. Inman, ${ }^{2,6}$ M. S. Kowitt, ${ }^{1,6}$ S. S. Meyer, ${ }^{1}$ L. A. Page, ${ }^{7}$ J. L. Puchalla, ${ }^{8}$ J. E. Ruhl, ${ }^{9}$ and R. F. Silverberg ${ }^{2}$ \\ Received 1999 February 3 ; accepted 1999 November 17
}

\begin{abstract}
We present new cosmic microwave background (CMB) anisotropy results from the combined analysis of the three flights of the first Medium Scale Anisotropy Measurement (MSAM1). This balloon-borne bolometric instrument measured about $10 \mathrm{deg}^{2}$ of sky at half-degree resolution in four frequency bands from 5.2 to $20 \mathrm{~cm}^{-1}$ with a high signal-to-noise ratio. Here we present an overview of our analysis methods, compare the results from the three flights, derive new constraints on the CMB power spectrum from the combined data, and reduce the data to total power Wiener-filtered maps of the CMB. A key feature of this new analysis is a determination of the amplitude of CMB fluctuations at $l \sim 400$. The analysis technique is described in a companion paper (L. Knox).

Subject headings: balloons - cosmic microwave background - cosmology: observations infrared: ISM: continuum
\end{abstract}

\section{INTRODUCTION}

The Medium Scale Anisotropy Measurement (MSAM) is a balloon-borne telescope and bolometric radiometer designed to measure the anisotropy in the cosmic microwave background (CMB) at angular scales near 0.5. The first two flights of MSAM1 (MSAM92: Cheng et al. 1994; MSAM94: Cheng et al. 1996) observed overlapping fields on the sky and demonstrated the reproducibility of the measurement. Two separate comparisons have been done that show consistency between these two flights (Inman et al. 1997; Knox et al. 1998). A third flight (MSAM95: Cheng et al. 1997) measured a nearby region of sky using the same observing method. This increased the sky coverage and sensitivity to the CMB anisotropy power spectrum. A second version of this instrument (MSAM2) with complementary frequency coverage has since been flown. This data set is still being analyzed.

\section{INSTRUMENT AND OBSERVATIONS}

The MSAM1 instrument has previously been described in detail (Fixsen et al. 1996). We give a summary here. The actively pointed gondola is comprised of a $1.4 \mathrm{~m}$ off-axis Cassegrain telescope with a multimode bolometric radiometer. A three-position chopping secondary throws the frequency-independent $\sim 0.5$ primary beam \pm 0.7 tangent to the local horizon at $2 \mathrm{~Hz}$. The four spectral channels at $5.7,9.3,16.5$, and $22.5 \mathrm{~cm}^{-1}$ each have bandwidth of $\sim 1.5$ $\mathrm{cm}^{-1}$. The detector outputs are synchronously sampled at $32 \mathrm{~Hz}$ : four times for each of four positions of the secondary mirror, for a total of 16 samples per chopper cycle. Tele-

\footnotetext{
${ }^{1}$ University of Chicago, 5640 South Ellis Avenue Chicago, IL 60637.

2 NASA Goddard Space Flight Center, Laboratory for Astronomy and Solar Physics, Code 685.0, Greenbelt, MD 20771.

${ }^{3}$ Fermilab, P.O. Box 500, Batavia, IL 60510.

${ }^{4}$ Global Science and Technology, Inc., NASA GSFC Laboratory for Astronomy and Solar Physics, Code 685.0, Greenbelt, MD 20771.

${ }^{5}$ Raytheon ITSS, NASA/GSFC Laboratory for Astronomy and Solar Physics, Code 685.0, Greenbelt, MD 20771.

${ }^{6}$ Stanford Research Systems, Sunnyvale, CA 94089.

${ }^{7}$ Princeton University, Princeton, NJ 08544.

8 Department of Physics and Astronomy, University of Pennsylvania, Philadelphia, PA 19104.

${ }^{9}$ University of California at Santa Barbara, Santa Barbara, CA 93106.
}

scope pointing is controlled with a star camera and gyroscope. The configuration of the gondola superstructure was changed between the 1992 and 1994 flights to reduce possible reflection of ground radiation. The improved configuration remained for MSAM95.

All three flights were launched from the National Scientific Balloon Facility in Palestine, Texas. The observing method is a slow azimuth scan of a region crossing the meridian above the north celestial pole. For a period of 20 minutes the scan center tracks a fixed spot on the sky as the Earth rotates. Afterward, an overlapping region is scanned. MSAM92 and MSAM94 observed at declination $\delta=$ +82.0 , MSAM95 at $\delta=+80.5$. The flights observed between right ascensions 14.2 and 19.5. The lower declination of MSAM95 required a faster scan rate because of the increased sky motion. The sky coverage of all the MSAM1 flights is shown in Figure 1.

\section{DATA REDUCTION}

The data from each of the three flights of MSAM1 are independently reduced in the same manner. We outline the process here.

1. Spikes caused by cosmic rays are removed from the time stream by a filtering and peak-detecting technique that results in the deletion of $5 \%-10 \%$ of the data. Samples are also lost due to spurious electrical pickup and telemetry dropouts. For each of these cuts, a full chopper cycle is deleted. The total loss is between $10 \%$ and $30 \%$ of the raw data.

2. The detector time streams are demodulated in two ways, each resulting in an independent instrumental beam pattern and corresponding instrumental window function. If $T_{L}, T_{C}$, and $T_{R}$ are the sky temperature at the left, center, and right positions of the beam during a chopper cycle, the single difference demodulation is $T_{R}-T_{L}$, making an antisymmetric beam pattern, while the double difference is $T_{C}-\left(T_{L}+T_{R}\right) / 2$, making a symmetric beam pattern. Optimum weighting for the demodulations are determined from Jupiter observations. The instrument noise is uncorrelated between the two demodulations.

3. The data are calibrated using scan and raster observations of Jupiter. The brightness temperature of Jupiter was found by cross-calibrating Jupiter with Mars with the 


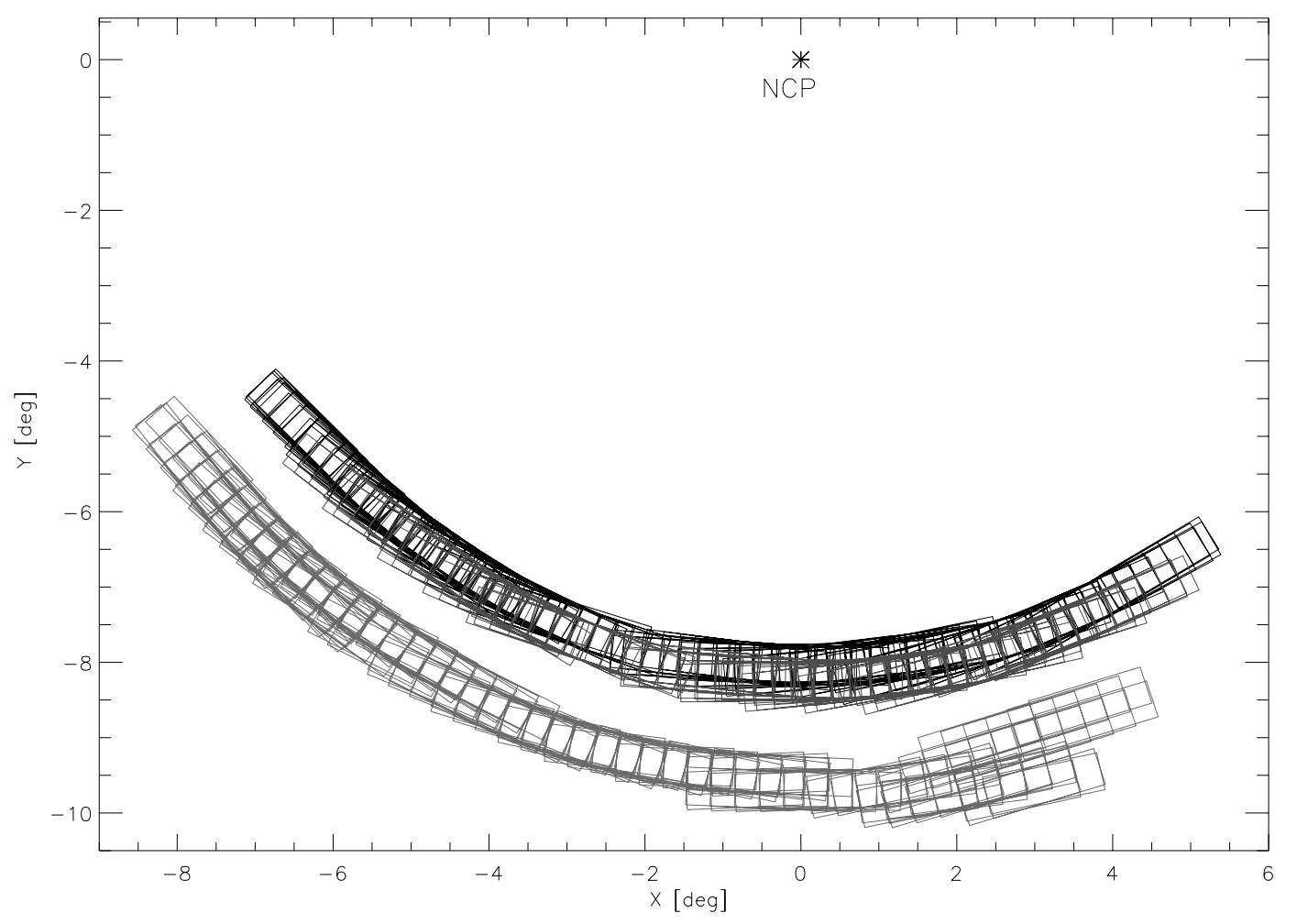

FIG. 1.-Locations in flat sky coordinates for the 1010 MSAM1 points. The boxes show the relative twist of the beam pattern during the observation. The upper row of points come from the overlapping flights, 2 years apart, of MSAM92 and MSAM94. The lower row are the MSAM95 points.

MSAM instrument during the 1992 flight (Goldin et al. 1997). Of the two models presented in that paper, we use the temperatures based on the "Rudy" model (Rudy et al. 1987). The error in the calibration is estimated to be $5 \%$, dominated by the uncertainty in the Jupiter temperature.

4. The Jupiter raster observations, performed during each flight, are the basis for the high-fidelity determination of the beam pattern for each demodulation. Beam pattern uncertainties are dominated by uncertainties in the reconstruction of the pointing. Pointing uncertainties are less than 2.5 .

5. The estimate of the instrument noise is determined from the variance in $100 \mathrm{~s}$ segments of the demodulated data after the removal of a slowly drifting offset. The offset ranges from 1 to $6 \mathrm{mK} R-J$ in MSAM94 and MSAM95 with an offset of $10 \mathrm{mK} R-J$ in all channels in MSAM92. The drift in the offset is small compared to its value. Because the removal of the offset correlates the noise on timescales longer than the detector time constant, the remainder of the data reduction incorporates the full noise covariance matrix.

6. The data are binned according to both the position on the sky and the twist of the demodulated beam pattern during each complete chopper cycle. The bin size for the twist dimension is determined by defining a "binning degradation factor", $\mathrm{BDF}=\left(\sigma^{2}+\left\langle\delta^{2}\right\rangle\right)^{1 / 2} / \sigma$, where $\sigma$ is the estimated instrument noise and $\left\langle\delta^{2}\right\rangle=\int\left[\mid B_{1}(k)-\right.$ $\left.\left.B_{2}(A \boldsymbol{k})\right|^{2} C_{\mathrm{cdm}}(\boldsymbol{k})\right] d \boldsymbol{k}$ is an estimate of the expected error in the estimate of the signal due to the twist bin size. The term $\left\langle\delta^{2}\right\rangle$ is determined using the standard cold dark matter correlation function $C_{\mathrm{cdm}}(\boldsymbol{k})$ convolved with beam patterns, $B_{i}(\boldsymbol{k})$ twisted with respect to each other by the rotation matrix $A$. A similar construction is used to define the BDF for the spatial binning. The BDF can be thought of as the factor by which the sensitivity of the data set is decreased due to the choice of bin size. The bin sizes are chosen to hold the BDF to values less than 1.1. This results in $5^{\circ}$ twist bins and $14^{\prime}$ bins in sky position.

7. The calibrated data are analyzed to provide measurements of brightness in the four spectral channels as a function of bin. The linear combination of the spectral channels, which minimizes the sensitivity of galactic dust foreground and matches the signature of a CMB thermal fluctuation over the spectral range of the instrument channels, is found and an estimate of CMB anisotropy and dust optical depth for each bin is produced. This is done by fitting the data for each bin in the four channels to a two-parameter model of sky and dust.

\section{COMPARISON OF MSAM92 AND MSAM94}

The overlapping regions of MSAM92 and MSAM94 (see Fig. 1) are used to compare their estimated sky signals. This can place a limit on how much of the signal could be attributed to instrumental artifacts or other local contamination. While straightforward in principle, a simple comparison is not possible despite the large degree of overlap. The beam centers for each sample do not line up perfectly and, because of the twist dimension in the binning, there are few bins that are populated in both flights. In Inman et al. (1997) the bin size was expanded over the criterion in the previous section, and those bins with sufficient data were differenced. With rather reduced sky coverage, Inman found no signal in the differenced data. 
An alternative procedure for comparing the two measurements has been previously reported in Knox et al. (1998). Here the most likely signal in the 1992 data is calculated by applying a Wiener filter to the 1992 observations. This signal is then compared to the most likely signal in the 1992 data given by the 1994 data by applying the appropriate Wiener filter to the 1994 data. In Figure 2 we see that the two data sets predict very similar signals for the 1992 data set using either the 1992 or 1994 data.

We quantify "very similar" by use of the likelihood ratio statistic. The two hypotheses are (1) the signals are correlated as one would expect (given the two sampling strategies and an assumed power spectrum) and (2) the signals are uncorrelated between data sets. We use the natural log of the likelihood ratio statistic, which is a quadratic operator on the data denoted by $\beta .{ }^{10}$ For the 1992 and 1994 data sets Knox et al. (1998) have found $\beta=12.8$, which means that

${ }^{10}$ Also see Tegmark (1998) on the optimization of quadratic comparison statistics. hypothesis 1 is $e^{12.8}$ times more likely than hypothesis 2 . A frequentist interpretation of $\beta$ is given by calculating the expected mean and standard deviation of the statistic under the different hypotheses. The result is $15.0 \pm 4.1$ (hypothesis 1) and $-58.4 \pm 27.4$ (hypothesis 2). This analysis is in agreement with Inman et al. (1997) that it is extremely unlikely that the data sets are caused by a signal that is uncorrelated between experiments. Based on these analyses, we conclude that the signal comes predominantly from the sky and not from the instrument or local environment.

\section{LIKELIHOOD ANALYSIS}

The data set from the three flights of MSAM has been reduced to 505 measurements of the CMB sky for each of the two demodulations. We model this data, $d$, as due to signal and noise,

$$
d_{i}=s_{i}+n_{i}
$$

where $i$ runs from 1 to 505 over the single-difference demodulation and from 506 to 1010 over the doubledifference demodulation, and the signal, $s$, is related to the

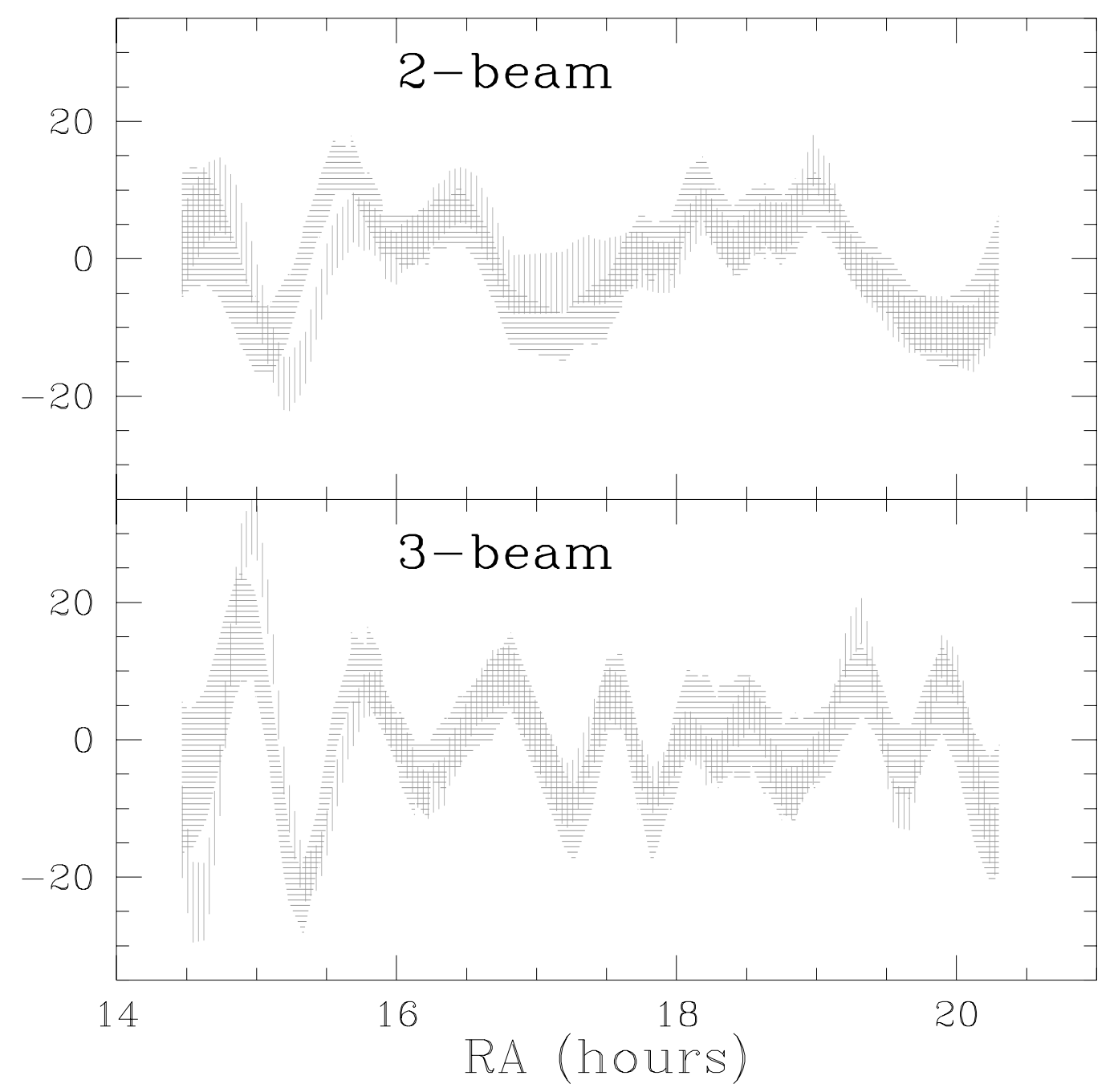

FIG. 2.-Most likely signal in 1992 data set, given the COBE-normalized standard CDM power spectrum and the 1992 data (vertical lines) or the 1994 data (horizontal lines). The shaded area is the $68 \%$ confidence region. Single-difference (or "2-beam") data is shown in the top panel, double-difference (or "3-beam") in the bottom panel. 
true temperature field, $T$, by

$$
s_{i}=\int_{\Omega} B(x-) T(x) d x .
$$

Here, $B(\boldsymbol{x})$ is the (single- or double-difference) beam map, and $\boldsymbol{x}_{i}$ specifies the pointing. We assume that both the signal and noise are Gaussian-distributed with zero mean with covariance matrices, which we denote by $S_{i j}=\left\langle s_{i} s_{j}\right\rangle$ and $N_{i j}=\left\langle n_{i} n_{j}\right\rangle$.

The noise covariance matrix, $N$, is block-diagonal with each block representing the noise correlations of a single demodulation from a single flight. The noise covariance matrix is singular due to the independent offset removals from each of the three flights (two each in MSAM92 and MSAM94). This constraint must be explicitly projected out of the data, which we do with a singular value decomposition (SVD) inversion of $N$.

The signal covariance matrix is linearly related to the angular power spectrum of the temperature field, $C_{l}$. The likelihood of this power spectrum, given the data, noise matrix, and our assumptions of Gaussianity is

$$
L\left(C_{l}\right)=\frac{e^{-1 / 2 d C^{-1} d^{T}}}{(2 \pi)^{N / 2} \sqrt{\operatorname{det} C},}
$$

where $\boldsymbol{d}$ is the $n$-element vector of observations and $C=$ $\left[S\left(C_{l}\right)+N\right]$ is the $(n \times n)$ covariance matrix of the observations. We use this likelihood to place limits on the power spectrum of fluctuations.

For this analysis we parameterize the theoretical signal covariance matrix, $S$, with the power spectrum, $\mathscr{C}_{l} \equiv l(l+1) C_{l} /(2 \pi)$, broken into bands denoted by $B$,

$$
\mathscr{C}_{l}=\sum_{B} \chi_{B(l)} \mathscr{C}_{B},
$$

so that within each band $B, \mathscr{C}_{l}=\mathscr{C}_{B}=$ constant. The sum runs over the bands in $l$-space with

$$
\chi_{B(l)}=\left\{\begin{array}{ll}
1 & l_{<}(B)<l<l_{>}(B) \\
0 & \text { otherwise }
\end{array} .\right.
$$

This parameterization of $\mathscr{C}_{l}$ is completely general and its usefulness will become apparent below.

The calculation of the likelihood requires the inversion of the $(n \times n)$ covariance matrix $C$. It has been shown (Bond 1994; Tegmark, Taylor, \& Heavens 1997; Bunn \& White 1997; Bond \& Jaffe 1997, p. 197) that a substantial reduction in the rank of $C$ can be achieved by working in the signal-to-noise $(\mathrm{S} / \mathrm{N})$ eigenmode basis. This is true even in a high $\mathrm{S} / \mathrm{N}$ case like that of MSAM1. For this data set, we achieve a compression by a factor of 1.8 in the rank of $C$ by ignoring modes with $\mathrm{S} / \mathrm{N}$ of less than 0.03 . Working in the $\mathrm{S} / \mathrm{N}$ eigenmode basis has the added benefit of automatically projecting out the eigenmodes associated with the offset removal. Thus, only one initial SVD of the covariance matrix is required (to zero the infinite eigenvalues). Inversions of the covariance matrix in the $\mathrm{S} / \mathrm{N}$ eigenmode basis are then done using faster methods such as Cholesky decomposition.

\section{THE FLAT BAND POWER}

As has been done previously for the individual data sets, we calculate "flat band powers" for each demodulation. That is, we assume the entire power spectrum is flat with amplitude $\mathscr{C}_{l}=\mathscr{C}_{B}$ and calculate the likelihood of this amplitude. Table 1 gives the flat band powers (maximum
TABLE 1

FLAT BAND-POWER EstimateS FOR MSAM1 (IN $\mu \mathrm{K}$ )

\begin{tabular}{ccc}
\hline \hline Flight & Single Difference & Double Difference \\
\hline MSAM92..... & $48 \pm 11$ & $54 \pm 10$ \\
MSAM94..... & $35 \pm 6$ & $45 \pm 9$ \\
MSAM95...... & $51 \pm 7$ & $56 \pm 7$ \\
All three ....... & $47 \pm 5$ & $53 \pm 5$ \\
\hline
\end{tabular}

likelihood values of $\sqrt{\mathscr{C}_{B}}$ ) for the three flights of MSAM1 for the single and double difference demodulations. The error bars indicate where the likelihood falls to $e^{-1 / 2}$ of the maximum.

\section{RADICAL COMPRESSION}

Flat band powers, together with a window function, have traditionally been the main results of $\mathrm{CMB}$ experiments. When taken together, they are the raw ingredients for constraining the power spectrum and cosmological parameters.

The parameters, $a_{p}$, are found by minimizing the $\chi^{2}$, where

$$
\chi^{2}=\sum_{B}\left[\sum_{l} W_{l}^{B} / l \mathscr{C}_{l}\left(a_{p}\right)-\mathscr{C}_{B}\right] / \sigma_{B}^{2},
$$

where $B$ runs over different data sets, $\mathscr{C}_{B}$ and $\sigma_{B}$ are the band powers and their standard errors, respectively, and $W_{l}^{B}$ is the band-power window function, which when summed over the power spectrum, $\mathscr{C}_{l}$, gives the theoretical prediction for the band power, $\mathscr{C}_{B}$. Although we do not do so here, the band-power window function is often assumed to be proportional to the variance window function, $W_{l}^{V}$, with proportionality constant chosen so that $\sum_{l} W_{l}^{V} / l=1$. The variance window function is defined so that $\sum_{l} W_{l}^{V} / l \mathscr{C}_{l}$ gives the expected signal contribution to the variance. The parameters, $a_{p}$, could be cosmological parameters (e.g., $\Omega_{b}$, $\Omega_{\Lambda}, H_{0}$, etc.) or parameters from a phenomenological power spectrum.

\subsection{Problems with Flat Band Powers ...}

Use of the flat band powers as a form of radical compression has the following drawbacks:

1. The actual sky power spectrum is not flat.

2. The band-power window function appropriate for equation (6) is, in general, not proportional to the variance window function as is often assumed.

3. The method provides no estimate of the correlation between the errors in the estimates of $\mathscr{C}_{B}$ from different demodulations.

4. The constraints on the parameters are not Gaussian, even though this assumption is implicit in the $\chi^{2}$ minimization.

Problems 1 and 3 are well-known deficiencies of the band power approach. Problem 4 has been previously emphasized (Bond, Jaffe, \& Knox 2000), where an approximate solution was given. Here we focus on problem 2 which has been discussed in Bond et al. (2000). We illustrate the potential severity of the problem with an extreme example. Consider a total power-mapping experiment with angular resolution of FWHM $=30^{\prime}$ which has measured a $5^{\circ} \times 5^{\circ}$ patch of the sky. The variance window function for this experiment is $W_{l}^{V}=\exp \left(-l^{2} \sigma_{b}^{2}\right)$, where $\sigma_{b} \simeq \mathrm{FWHM} /$ 2.355. Note that this window peaks at low $l$, indicating 
that the experiment is most sensitive to fluctuations on very large angular scales. However, the data set is not actually sensitive to the lowest spatial frequencies at all. The problem lies in having ignored the off-diagonal terms. The variance window function is equal to the band-power window function only if the data points are all far apart on the sky so that $S_{i j}$ is diagonal. For the example given, correlations between the points on the sky are making the data set insensitive to fluctuations on large scales. The variance window function is unaffected by these correlations, so we get a nonsensical result. For most actual data sets the problem is not quite so severe, but this example illustrates the potential pitfall.

\section{2. ... and Solutions}

Solutions have been found to all of these problems (Bond, Jaffe, \& Knox 1998; Bond et al. 2000; Knox 1999). Here we briefly review them.

Problem 1 can be solved by breaking the power spectrum into several bands as in equation (4), and then finding the amplitudes of these bands, $\mathscr{C}_{B}$, that maximize the likelihood. We find this maximum by iterative application of a quadratic estimator, as has been done for COBE DMR (Bennett et al. 1996) and the Saskatoon (Netterfield et al. 1997) data in Bond et al. (1998), and on simulated MAP data (Oh, Spergel, \& Hinshaw 1999). By calculating the covariance matrix of the set of $\mathscr{C}_{B}$, we also solve problem 3 .

Because physical power spectra are not actually flat across these bands, we need a means of taking a general power spectrum, $\mathscr{C}_{l}$, and turning it into a prediction for $\mathscr{C}_{B}$. In other words, we need to be able to calculate the expectation value of $\mathscr{C}_{B},\left\langle\mathscr{C}_{B}\right\rangle$, under the assumption that the power spectrum is $\mathscr{C}_{l}$. This relationship is specified by the band-power window function,

$$
\left\langle\mathscr{C}_{B}\right\rangle=\sum_{l} W_{l}^{B} / l \mathscr{C}_{l} .
$$

Taking equation (7) as the definition of the band-power window function, Knox (1999) has shown how it can be calculated from the signal and noise covariance matrices and the derivatives of $S$ with respect to $\mathscr{C}_{l}$. Taking into account all off-diagonal terms, this prescription for $W_{l}^{B}$ solves problem 2 .

We could remove the need for window functions by making the bands very narrow since sufficiently narrow bands ensure that the sensitivity to each $\mathscr{C}_{l}$ within the band is approximately independent of $l$. However, making the bands too narrow would increase the nonGaussianity, exacerbating problem 4 because the likelihood of more tightly constrained broad bands is better approximated by a Gaussian. Therefore, the bands must be broad enough to have significant constraints on their amplitudes. For MSAM, this condition makes the bands sufficiently broad that the sensitivity to $\mathscr{C}_{l}$ varies significantly across the bands, necessitating the use of a separate window function for each band.

Finally, if we adopt the Bond et al. (2000) prescription for problem 4, which requires calculation of a "log-normal offset," $x_{B}$, for each $\mathscr{C}_{B}$, we have solutions to all four problems. Although these solutions are not exact, they do represent a significant improvement over the usual flat band-power method.

It is not necessary to break the power spectrum into bands to obtain parameter estimates from the observations. However, this approach aids the comparison of different experiments with a minimum of theoretical assumptions, as well as easing the comparison of experimental results with theory. By following the above procedure for powerspectrum estimation, the full weight of an experiment is made available in an easily tractable form for the kind of parameter estimation outlined in equation (6).

\subsection{The Application to MSAMI Data}

The MSAM1 data sets are a prime example of the limitations of the flat band-power method. The MSAM1 data have high $\mathrm{S} / \mathrm{N}$ and are heavily sample-variance limited when using standard estimators of the flat band power. We now use the radical compression methods outlined above to probe regions of $l$-space ignored by our previous reduction to flat band powers.

The difference between the band-power window functions, $W_{l}^{B}$, and the variance window functions, $W_{l}^{V}$, is shown in Figure 3. The plot shows the window functions for each demodulation of the $3 \mathrm{yr}$ data for a single band covering all $l$. Notice that the $W_{l}^{B}$ band-power window functions show more response at high $l$ than the $W_{l}^{V}$ 's. This is because the dense sampling and high $\mathrm{S} / \mathrm{N}$ of the data set yield information on angular scales smaller than the beam size. Again, this information is in the off-diagonal components of the covariance matrix - underscoring the need for experiments to track the full noise covariance matrix in the data reduction.

We plot the window functions for the individual demodulations only to make the point that $W_{l}^{B} \neq W_{l}^{V}$, even though their equality is often assumed. In the analysis we describe below, we do not treat the double-difference data sets and single-difference data sets separately; the very significant correlations between them are included.

For this analysis, we break up the $l$-space coverage into

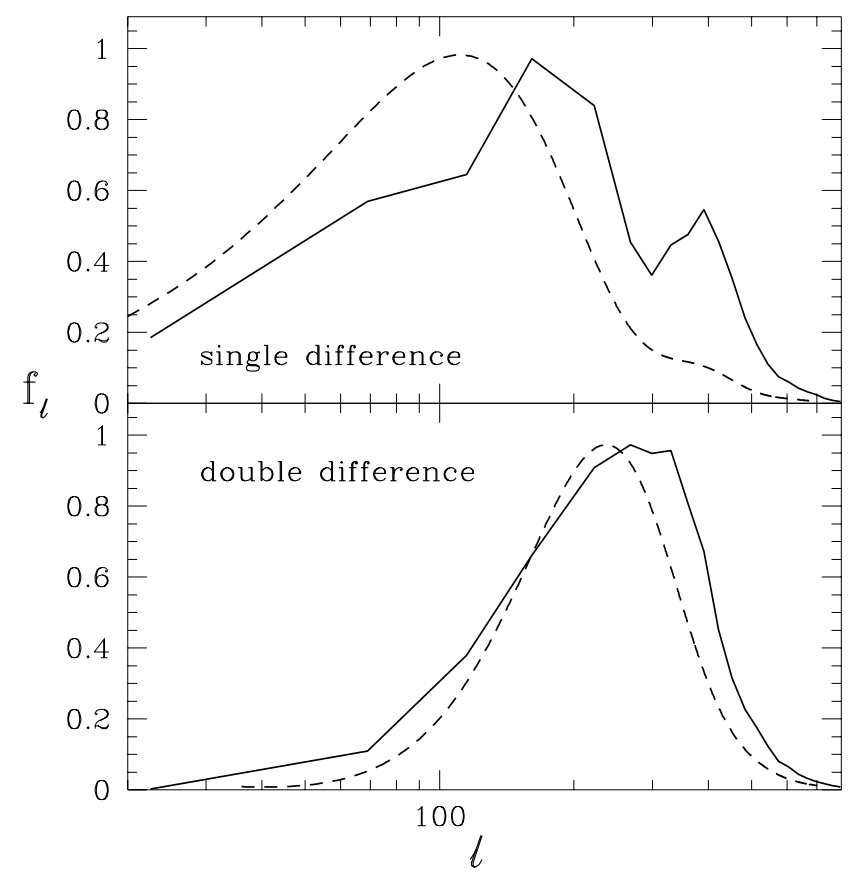

Fig. 3.-Band-power window functions (solid lines) and variance window functions (dashed lines) for the single- and double-difference demodulations $\left(f_{l} \equiv W_{l} / l\right)$. 


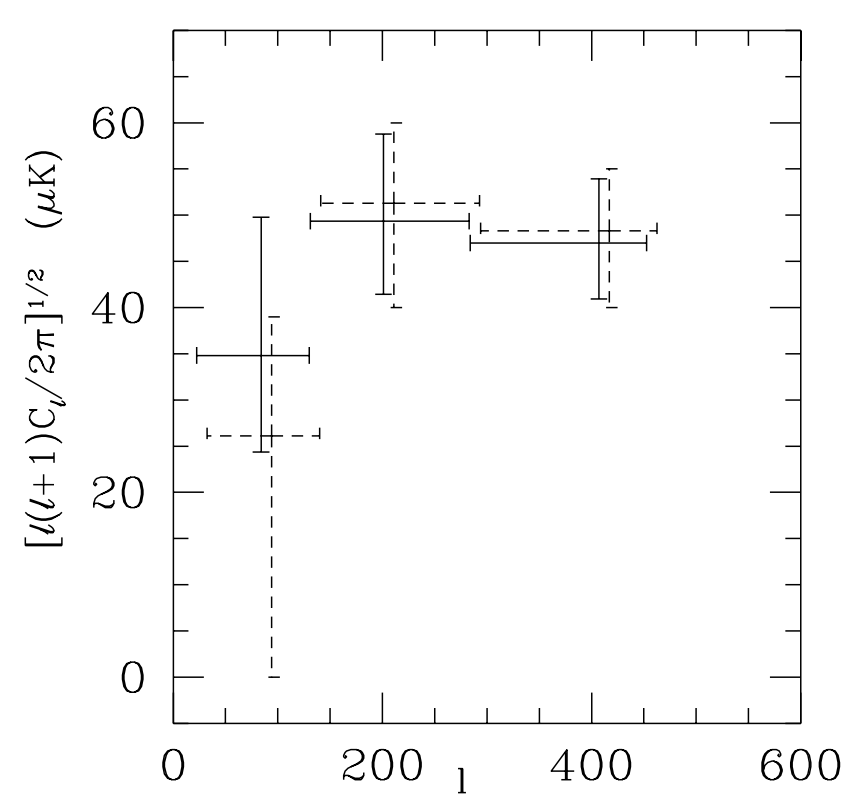

FIG. 4.-MSAM band-power estimates. The solid lines give the estimates of the power in the three bands calculated directly from the demodulated data and recorded in Table 2. Error bars due to the calibration uncertainty are not included. Similar results (dashed lines) are achieved by analyzing a total-power map of the CMB temperature, which is constructed from the demodulated data (see $\S 8$ ).

three wide bands and allow $\mathscr{C}_{B}$ to vary in each. In line with the discussion above, we choose the three bands such that each has enough weight to produce an interesting constraint on the power spectrum. The $l$-ranges for the three bands chosen are 39-130,131-283, and 284-806.

In Figure 4 we plot the three power spectrum estimates, $\hat{\mathscr{C}}_{B}$, from the combined $3 \mathrm{yr}$ of data. The central points are located in $l$-space at $l_{\text {eff }}$, which we define as the average value of $l$ over $W_{l}^{B} / l$ for that band. We include a horizontal bar from $l_{-}$to $l_{+}$. For the middle band, these are taken to be simply the beginning and end of the band $\left(l_{-}=l_{<}=\right.$ $131, l_{+}=l_{>}=283$ ). For the far ends of the two outer bands, we take them to be where $W_{l}^{B} / l$ falls to $e^{-1 / 2}$ of maximum. Similar results (dashed lines) are achieved by analyzing a total-power map of the CMB temperature, which is constructed from the demodulated data. We take the good agreement between these two nearly independent analysis techniques as strong evidence that we have calculated the complicated signal covariances correctly. We will discuss this procedure in $\S 8$. (See Table 2 for power spectrum estimates.)

While the $\hat{\mathscr{C}}_{B}$ are not independent, their correlation coefficients are fairly small. The correlation between bands 1 and 2 is -0.18 , between bands 1 and 3 is -0.024 , and between bands 2 and 3 is -0.29 . The error bars shown in

TABLE 2

POWER SPECTRUM ESTIMATES FROM MSAM1

\begin{tabular}{crcc}
\hline \hline$l_{-}$ & $l_{\text {eff }}$ & $l_{+}$ & $\begin{array}{c}\sqrt{\hat{\mathscr{C}}_{B}} \\
(\mu \mathrm{K})\end{array}$ \\
\hline $39 \ldots \ldots$ & 84 & 130 & $35_{-11}^{+15}$ \\
$131 \ldots \ldots$ & 201 & 283 & $49_{-8}^{+10}$ \\
$284 \ldots \ldots$ & 407 & 453 & $47_{-6}^{+7}$ \\
\hline
\end{tabular}

NoTE.-Calibration uncertainty not included.
Figure 4 are the result of marginalizing over the power in the other bands. Under the assumption that the other bands are fixed, the error on the band in question is less than $5 \%$ smaller.

The power spectrum estimates, $\hat{\mathscr{C}}_{B}$, their weight matrix, $F_{l l^{\prime}}$, window functions, $W_{l}^{B}$, as well as log-normal offsets, $x_{B}$ (see Bond et al. 2000) are available at $\mathrm{NASA}^{11}$ and University of Toronto ${ }^{12}$ websites, which include similar information from other CMB anisotropy data sets.

\section{CMB MAPS}

A useful check of our power spectrum results can be made by analyzing a map made from the demodulated data as opposed to directly from the demodulated data as we have done above. We begin constructing this map by recognizing that equations (1) and (2) can be combined and rewritten in matrix form as

$$
d=B T+n,
$$

where $B$ and $n$ are matrices denoting the beam map and noise, respectively, and $T$ is a vector of temperatures. With the assumption that the noise is Gaussian, with covariance matrix, $N$, the most likely value of $T$, given the data, $d$, is that which minimizes the $\chi^{2}$ :

$$
\chi^{2} \equiv(d-B T) N^{-1}(d-B T) .
$$

This minimum, which we denote by $\hat{T}$, is given by

$$
\hat{T}=\tilde{N} B N^{-1} d .
$$

This estimate of $T$ will be distributed around the true value due to noise, where the noise covariance matrix is

$$
\tilde{N} \equiv\langle(\hat{T}-T)(\hat{T}-T)\rangle=\left(B^{T} N^{-1} B\right)^{-1} .
$$

This map can be analyzed in the same manner as the demodulated data, with the advantage that the signal covariance matrix is now very simple to compute. Previously, calculating the signal covariance matrix required a four-dimensional integral for every covariance element. In this new "map basis," the signal covariance matrix simplifies to

$$
\left\langle T_{i} T_{j}\right\rangle=\sum_{l} \frac{2 l+1}{4 \pi} P_{l}\left[\cos \left(\theta_{i j}\right)\right] C_{l} .
$$

The price to pay for this simplicity is that the noise covariance, $\widetilde{N}$, is very complicated. We have done this analysis as a check of the calculations in $\S 7.2$. The results are shown in Figure 4 . The agreement is a strong argument that we have made no errors in what is a fairly elaborate and difficult calculation.

The map, $\hat{T}$, is extremely noisy and not visually useful. We can greatly reduce the noise by Wiener filtering, (e.g., Bunn, Hoffman, \& Silk 1996; Tegmark et al. 1997; Knox et al. 1998). The Wiener filter produces the most likely $T$, given not only the data, but also an assumed power spectrum for the signal. The Wiener filtered maps are shown in Figure 5.

\section{DISCUSSION}

Because the third band is derived by making measurements on scales on order of the beam size, we must ask what

\footnotetext{
${ }^{11} \mathrm{http}$ ://topweb.gsfc.nasa.gov

$12 \mathrm{http}: / /$ www.cita.utoronto.ca/knox/radical.html
} 


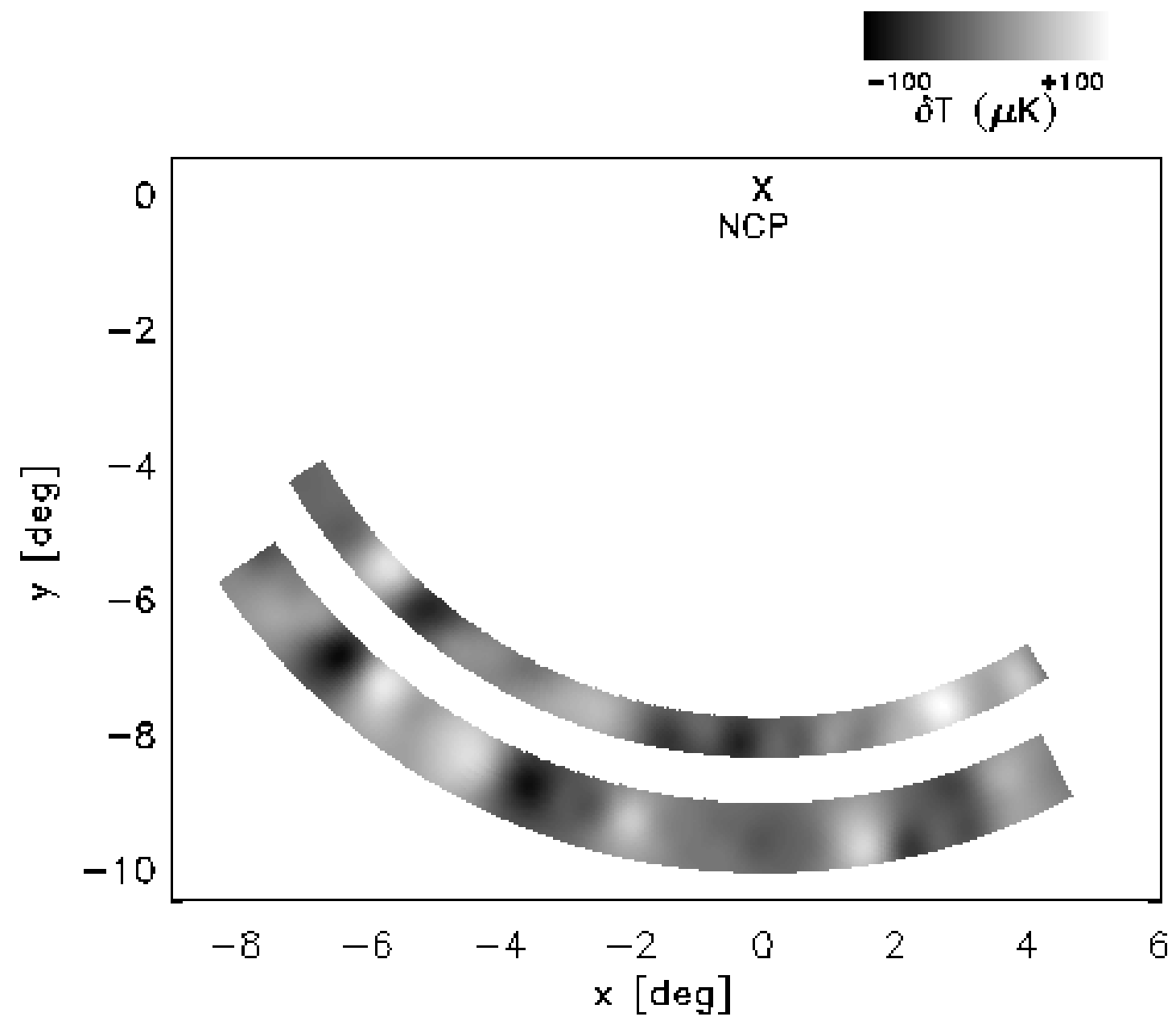

FIG. 5.-Map of the 3 years of data. Top region was covered by the 1992/1994 flight; bottom by 1995 flight. Note that the scan pattern filters out all of the low spatial frequencies from the map.

sensitivity the amplitude of $\hat{\mathscr{C}}_{B}$ has to the beam shape. For example, if the band sensitivity results from high frequency fluctuations in the measurements of the various $B_{i}(\vec{k})$, the estimation of the amplitude of $\mathscr{C}_{B}$ would be sensitive to the errors in determining the beam map. We address this question by performing a number of analyses of the $3 \mathrm{yr}$ of data. The first analysis (leading to the quoted values of $\hat{\mathscr{C}}_{B}$ here) is done using the six beam maps measured in the three flights. That is, MSAM92 data goes with the MSAM92 beam maps, MSAM94 data with MSAM94 beam maps, and MSAM95 data with MSAM95 beam maps. Since the beam maps from year to year differ slightly in overall shape due to variations in the assembly of the optical system, a second analysis is done using the beam maps measured during the MSAM92 flight for all three years of data. This is followed by repeating the analysis with the beam maps from MSAM95. ${ }^{13}$ Reanalyzing the entire data set in this way is taken to be the most pessimistic estimator of the effect of the beam on the third band. The differences between the beam maps include all the statistical errors of the beam maps, any errors in the raster observations themselves, and any changes introduced by the complete rebuild, realignment, and refocusing of the optical system and instrument con-

\footnotetext{
13 The MSAM92 and MSAM94 beam maps are similar enough to be swapped with no change in estimated signal.
}

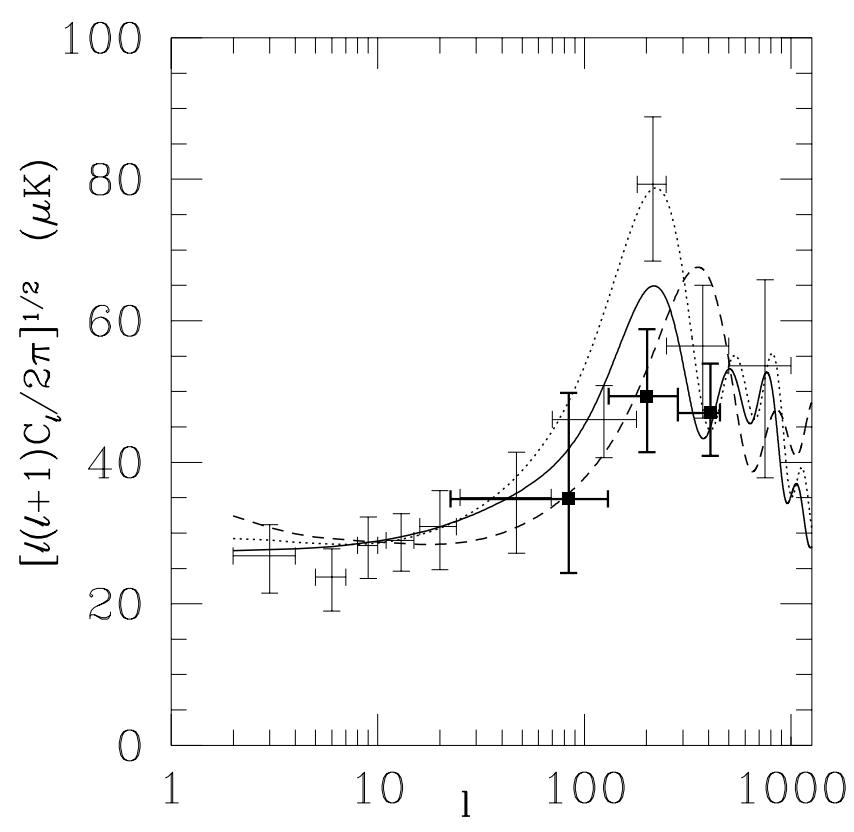

FIG. 6.-Dark error bars are the power spectrum constraints from the 3 year MSAM data set. The light error bars are the result of a fit of the power in 11 bands (as done in Bond et al. 2000) to all data available as of 1999 January, including the previously published MSAM1 points. The curves are standard CDM (solid curve), a flat lambda model (dotted curve), and an open model with $\Omega_{\text {curvature }}=0.6$ (dashed curve). 
figuration. We find that after accounting for the normalizations of the different data sets, there is no evidence for the third band being sensitive to the beam map choice.

To place our estimates of the power spectrum in context, we plot them with the predictions of several theoretical models as well as a fit of the power in 11 bands to all available data (based on Bond et al. 2000), including the previously published MSAM1 points.

There are several things to note from Figure 6. First, although the MSAM dataset by itself is consistent with a flat power spectrum, they do indicate more power at $l \sim 200$ and $l \sim 400$ then there is at $l \sim 10$ as determined by $C O B E$. Second, the power at $l \sim 200$ is less than that determined from the fit to all experiments. Including calibration uncertainty, this discrepancy is marginal (Dodelson \& Knox 1999). Finally, the constraint at $l \sim 400$ is a significant improvement upon others at this $l$.

\section{SUMMARY}

We have calculated new power spectrum estimates from the combined three flights of the MSAM1 instrument. The analysis technique used is an improvement over the standard flat band-power approach and includes all correlations in the data. In addition to power-spectrum estimates and their error covariance matrices, we have also provided the log-normal offsets and band-power window functions in order to improve "radical compression." The analysis yields a strong constraint on the power spectrum at $l \sim 400$, broadening the $l$-space coverage of the experiment into a theoretically very interesting region.

This work would not be possible without the excellent support we receive from the staff of the National Scientific Balloon Facility. Financial support was provided by the NASA Office of Space Science, under the theme "Structure and Evolution of the Universe." G. W. is supported by a National Research Administration fellowship. K. C. is supported by a Graduate Student Researcher Program fellowship.
Bennett, C. L., et al. 1996, ApJ, 464, L1

Bond, J. 1994, Phys. Rev. Lett., 74, 4369

Bond, J. R., \& Jaffe, A. H. 1997, in Proc. 31st Moriond Meeting, Microwave Background Anisotropies, Cosmic Parameter Estimation Combining Sub-Degree CMB Experiments with $C O B E$, ed. Bouchet, Gispert, Guilderdoni \& Van (Gif-sur-Yvette: Frontières)

Bond, J. R., Jaffe, A., \& Knox, L. 1998a, Phys. Rev. D, 57, 2117 $2000, \mathrm{ApJ}$, in press (astro-ph/9808264)

Bunn, E. F., Hoffman, Y., \& Silk, J. 1996, ApJ, 464, 1

Bunn, E. F., \& White, M. 1997, ApJ, 480, 6

Cheng, E. S., et al. 1994, ApJ, 422, L37 1996, ApJ, 456, L71

. 1997, ApJ, 488, L59

Dodelson, S, \& Knox, L. 1999, Phys. Rev. Lett., submitted (astro-ph/ 9603121)

\section{REFERENCES}

Fixsen, D. J., et al. 1996, ApJ, 470, 63

Goldin, A. B., et al. 1997, ApJ, 488, L161

Inman, C. A., et al. 1997, ApJ, 478, L1

Knox, L. 1999, preprint (astro-ph/9902046)

Knox, L., Bond, J., Jaffe, A., Segal, M., \& Charbonneau, D. 1998, Phys. Rev. D, 58, 083004

Netterfield, C. B., Devlin, M. J., Jarosik, N., Page, L., \& Wollack, E. J. 1997, ApJ, 474, 47

Oh, S., Spergel, D., \& Hinshaw, G. 1999, ApJ, 510, 551

Rudy, D. J., Muhleman, D. O., Berge, G. L., Jakosky, B. M., \& Christensen, P. R. 1987, Icarus, 71, 159

Tegmark, M., de Oliveira-Costa, A., Devlin, M., Netterfield, C., Page, L., \& Wollack, E. 1997, ApJ, 474, L77

Tegmark, M., Taylor, A., \& Heavens, A. 1997, ApJ, 480, 22 